\title{
Identificação dos níveis de stress em equipe de atendimento pré-hospitalar móvel
}

\author{
Identification of the levels of stress in a \\ pre-hospital mobile care team
}

\author{
Sandra Soares MENDES' \\ Luciane Ruiz Carmona FERREIRA' \\ Milva Maria Figueiredo De MARTINO
}

\section{Resumo}

Este estudo, de natureza descritiva, transversal e quali-quantitativa, teve como objetivo identificar a presença e os níveis de stress em equipe multiprofissional de atendimento pré-hospitalar. A pesquisa foi realizada na unidade de Serviço de Atendimento Móvel de Urgência da cidade de Poços de Caldas, Minas Gerais. Participaram da pesquisa 63 sujeitos de ambos os sexos, com idade média de 32,6 anos, abrangendo diversas categorias profissionais e diferentes turnos de trabalho. Os instrumentos utilizados foram ficha de caracterização sociodemográfica e Inventário de Sintomas de Stress de Lipp para Adultos. Foram encontrados os seguintes resultados: $31,7 \%$ dos sujeitos apresentavam sintomas de stress, 30,2\% dos quais na fase de resistência, com predomínio de sintomas físicos; 66,7\% não tinham outra atividade, sendo que 52,4\% desse grupo não apresentavam sintoma de stress (Teste Exato de Fisher $p=0,015$ ). Concluiu-se que os indivíduos apresentavam baixos níveis de stress e que se utilizavam de mecanismos cognitivos ou comportamentais para minimizá-lo e manter o equilíbrio interno.

Unitermos: Equipe de assistência ao paciente. Serviço médico de emergência. Stress.

\begin{abstract}
This study combined descriptive, cross-sectional and qualitative and quantitative aspects and aimed to identify the presence and levels of stress in a pre-hospital care unit. The study was performed at a Mobile Emergency Care unit in the city of Poços de Caldas, Minas Gerais. Sixty-three individuals of both sexes, with a mean age of 32.6, took part in the study, encompassing various professional groups and shift workers. The instruments used were: Record of socio-demographic characteristics and the Lipp's Stress Symptoms Inventory for Adults. The results were as follows: $31.7 \%$ of subjects showed symptoms of stress, $30.2 \%$ were in the resistance stage, with predominantly physical symptoms; $66.7 \%$ enjoyed no other activity, and of these $52.4 \%$ had no symptoms of stress (Fisher's exact test $p=0.015$ ). We conclude that the individuals displayed low levels of stress and they used cognitive or behavioral mechanisms to minimize stress and maintain internal balance.
\end{abstract}

Uniterms: Patient care team. Emergency medical service. Stress.

$\boldsymbol{\nabla \nabla v \boldsymbol { V }}$

1 Universidade Estadual de Campinas, Faculdade de Ciências Médicas, Departamento de Enfermagem. R. Tessália Vieira de Camargo, 126, Cidade Universitária Zeferino Vaz, 13083-887, Campinas, SP, Brasil. Correspondência para/Correspondence to: S.S. MENDES. E-mail: <sandras.mendes@hotmail.com>.

Agradecimento: à Coordenação de Aperfeiçoamento de Pessoal de Nível Superior, pelo apoio através da concessão de bolsa de doutorado à autora Luciane Ruiz Carmona Ferreira 
Para a Organização Mundial da Saúde (OMS), o stress tem sido considerado uma epidemia global, em virtude da constante atualização das informações, o que pode interferir na qualidade de vida dos sujeitos, resultando em prejuízos de ordem familiar, social, falta de motivação para atividades em geral, doenças físicas e psicológicas, além de problemas no trabalho (Lipp, 1996; Jacques, 2004).

Fato importante é que, independentemente do local de residência dos indivíduos, o stress pode estar presente e ser relacionado a diferentes agentes causadores, conforme verificado por Silva e Martinez (2005), em estudo realizado a fim de avaliar as diferenças em nível de stress entre sujeitos moradores da capital e do interior de São Paulo.

Na área da saúde, a terminologia stress foi utilizada pela primeira vez em 1936, pelo médico endocrinologista Hans Selye, que a definiu como uma resposta não específica do organismo diante de uma situação que ameace a homeostase, em que seja necessária uma mobilização para enfrentar o evento que causou a alteração em nível biopsicossocial (Lipp, 2003a; Selye, 1954).

O stress é um processo que exige a adaptação do organismo a uma condição externa ou interna, que de algum modo esteja alterando a percepção de bem-estar do indivíduo (Calais, 2003; Lipp, 2003b). As reações dos indivíduos frente ao stress são diferentes. Uma resposta básica ao stress é preparar o indivíduo para "lutar ou fugir". O grau de stress vivenciado não se relaciona somente com as situações que o causaram, mas também com o modo como o indivíduo o percebe e reage diante da situação estressora (Glassman \& Hadad, 2006).

O stress pode ocorrer de duas maneiras, a primeira de natureza aguda e muito intensa, mas que desaparece rapidamente; e a segunda de natureza crônica e não tão intensa, podendo ocorrer por tempo mais prolongado, sendo poucos os recursos utilizados pelo indivíduo para enfrentá-lo (Lipp, 1996). tuação de stress, surgem reações distintas que podem ser divididas nas seguintes fases:

1) Fase de alarme ou alerta: é a resposta inicial do organismo ao estressor, mobilizando uma resposta orgânica rápida para o enfrentamento. Podem ser obser200 vadas nesta fase várias reações fisiológicas, como sudo- rese excessiva, taquicardia, respiração ofegante. Ocorre também a face positiva do stress, com aumento do nível de atenção e velocidade na articulação de pensamentos, além de aumento na motivação e vontade de iniciar novos projetos (Lipp, 2003a).

2) Fase de resistência: ocorre aumento da capacidade de resistência do organismo, independentemente da permanência ou não do estressor, com uso de toda a energia e recursos disponíveis, podendo gerar sensação de desgaste idiopático, inclusive danos à memória. Nesse momento há adaptação do organismo, com a respiração, os batimentos cardíacos, a circulação e a pressão arterial voltando aos níveis anteriores (Lipp, 2003a).

3) Fase de quase exaustão: fase recentemente descoberta por Lipp (2000), caracterizada pelo enfraquecimento e incapacidade do indivíduo em resistir ou se adaptar ao estressor, podendo ocasionar leves problemas de saúde, que não o incapacitam (Lipp, 2003a).

4) Fase de exaustão: caracterizada pela impossibilidade de resistência ao estressor, em que o organismo é incapaz de eliminá-lo ou adaptar-se adequadamente, com surgimento de patologias orgânicas e psíquicas. Podem ser observados sintomas específicos dos órgãos afetados e da patologia que nele se instalar, podendo ocorrer enfarte, úlceras, psoríase, depressão e outros, ou até mesmo a morte em casos mais graves (Lipp, 2003a).

Quando o ambiente de trabalho é percebido como uma ameaça ao indivíduo, ocorre o stress no trabalho, com repercussões na sua vida pessoal e profissional (Martins, Bronzatti, Vieira, Parra \& Silva, 2000). Nos últimos anos, tem aumentado o interesse na literatura científica pelo estudo do stress no trabalho. Um dos motivos das pesquisas sobre o tema é o impacto negativo do stress ocupacional na saúde e no bem-estar dos trabalhadores e, consequentemente, no funcionamento e na efetividade das organizações (Cox, 1987; Paschoal \& Tamayo, 2004).

O que tem sido observado nas instituições de saúde é que elas utilizam de ferramenta para diminuir os custos, por meio da exploração dos trabalhadores, com aumento gradativo da jornada de trabalho e redução da mão de obra. A redução no contingente de pessoal reflete diretamente no trabalho dos profissionais que se mantêm na instituição, pois eles são coagidos a 
aumentar a sobrecarga na execução de suas atividades, desencadeando situações de tensão no ambiente de trabalho, com significativo comprometimento da assistência prestada ao paciente (Sears, Murphy, Sinclair, Davidson \&Wang, 2008).

O stress ocupacional está determinado pela percepção que o trabalhador tem das necessidades do ambiente de trabalho e de sua habilidade para enfrentá-las (Santos, Oliveira \& Moreira, 2006). As fontes que geram stress são sentidas e interpretadas pelas pessoas de maneira diferente, devendo-se considerar as características de cada indivíduo e sua capacidade de interagir com os estressores do ambiente exposto, com variação dos níveis de stress (Martins et al., 2000).

Níveis altos e contínuos de stress podem desencadear doenças físicas, gerar quadro de esgotamento emocional com sentimentos negativos, como pessimismo, atitudes desfavoráveis em relação ao trabalho, comportamento alterado com colegas, informações novas ignoradas e resolução superficial de problemas (Lipp \& Tanganelli, 2002; Pafaro \& De Martino, 2004).

Normalmente, para lidar com o stress, o indivíduo pode buscar respostas comportamentais e cognitivas (conhecidas como processo de enfrentamento da situação - coping). Existem dois tipos de estratégias: as que são concentradas no problema e as que são concentradas na emoção. Quando o enfrentamento está centrado no problema, o indivíduo tenta lidar de forma direta com a situação e testa maneiras de resolvê-la, como falar sobre ações de confronto direto e indireto. Quando o enfrentamento está voltado para a emoção, o indivíduo utiliza estratégias emocionais ou cognitivas que mudam a maneira de ver a situação estressante, distanciando-se do problema e procurando evitá-lo (Bachion, Peres, Belisário \& Carvalho, 1998; Lazarus \& Folkman, 1984; McGowan, Gardner \& Fletcher, 2006).

Todas as profissões e atividades podem gerar certo grau de stress, embora atividades relacionadas aos cuidados de saúde sejam caracterizadas por alguns estressores que são o resultado de um intenso compromisso com o cuidado. Assim, o compromisso com a vida, o relacionamento e empatia emocional com o paciente, assim como as características da instituição, podem pôr em risco constante os trabalhadores da área de saúde (Camponovo Meier \& Morín Imbert, 2000).
O elevado nível de stress enfrentado pelos profissionais de saúde ocorre pela exposição contínua a dificuldades próprias de seu cotidiano de trabalho, como o sofrimento, a dor, a morte, as longas jornadas de trabalho, o relacionamento com paciente e colegas, e as normas institucionais (Zaldúa \& Lodieu, 2000).

\section{Serviço de atendimento móvel de urgência}

Conforme a Política Nacional de Atenção às Urgências, o Serviço de Atendimento Móvel de Urgência (SAMU) é o componente principal para garantir a qualidade de atendimento no Sistema Único de Saúde (SUS). O socorro ocorre em circunstâncias de agravo urgente, com intervenção no local da ocorrência, com atendimento rápido, seguro e eficaz, com meios adequados e garantia de acesso aos sistemas de saúde. O nível de competência dos profissionais e o trabalho em equipe garantem de forma direta a qualidade da assistência (Brasil, 2004a; Brasil, 2006).

O SAMU foi criado no Brasil pela Portaria no 1.864 GM/MS, de 29 de setembro de 2003. O Regulamento Técnico dos Sistemas Estaduais de Urgência e Emergência foi instituído pela Portaria GM/MS 20.048, em 5 de novembro de 2002. A Portaria no 1.863 GM/MS, de 29 de setembro de 2003, instituiu a Política Nacional de Atenção às Urgências, e, de acordo com seu artigo 30, a organização de redes loco-regionais de atenção integral às urgências (Brasil, 2002; Brasil, 2003).

O SAMU é um serviço que implementa a Assistência Pré-Hospitalar (APH) no SUS, acionado por discagem rápida pelo número nacional 192, em que a ligação é gratuita. A regulação é através de Centrais de Regulação de Urgência. Técnicos da Central de Regulação fazem o atendimento das ligações, que transferem rapidamente para o médico regulador, o qual faz um diagnóstico da situação para que se inicie o atendimento imediatamente. A orientação das ações então são passadas ao paciente ou pessoa que realizou o chamado (Brasil, 2004b; Brasil, 2006).

O trabalho realizado pelos profissionais do atendimento pré-hospitalar móvel não pode ser previsto; quanto maior for a incerteza do evento, maior poderá ser a geração de sentimentos de ameaça, exigindo dos profissionais competência e esforço para salvar a vida, com tomada de decisão rápida e eficaz (França \& 
Rodrigues, 2002). Pela complexidade das situações de urgência e emergência com as quais os profissionais se deparam em sua rotina de trabalho, destaca-se a presença de estressores que podem prejudicar o trabalho do profissional ou da equipe (De Gasperi \& Radünz, 2006).

Segundo Murta e Tróccoli (2007), bombeiros e profissionais envolvidos em atendimento de emergência são mais susceptíveis a desenvolver stress no trabalho. Além dos fatores psicológicos, esses indivíduos estão expostos também a fatores biológicos que apresentam característica estressante.

Uma circunstância ou experiência que gera sentimentos de medo, ansiedade, tensão ou ameaça é um estressor. A sobrecarga de trabalho mental, físico e psíquico à qual os profissionais de emergência estão submetidos, assim como a gravidade dos pacientes, a possível mudança no estado geral da vítima, a morte, o deslocamento da ambulância, o tráfego, os locais das ocorrências, o atendimento em si, a família da vítima e a expectativa do envio podem ser considerados como geradores de stress, exigindo equilíbrio dos profissionais na tomada de decisões, a fim de que o atendimento seja adequado (Stacciarini \& Tróccoli, 2000).

O trabalho em turno é outro fator gerador de stress. As jornadas de trabalho noturnas podem levar ao desconforto e mal-estar, com alterações do ritmo biológico circadiano em relação à alternância sono-vigília, da temperatura corporal, dos níveis hormonais, além de distúrbios digestivos, nervosos e de personalidade, repercutindo no convívio familiar e social (De Martino \& Cipolla-Neto, 1999).

Dessa forma, o estudo teve como objetivo identificar a presença e os níveis de stress em equipe multiprofissional de atendimento pré-hospitalar móvel, para conhecer a realidade vivida por esses profissionais, a fim de oferecer subsídios para elaboração de programa de atenção à sua saúde. quali-quantitativa, foi desenvolvido em uma unidade de SAMU, na cidade de Poços de Caldas (MG). A unidade contava com 66 funcionários, divididos nos cargos de médico, enfermeiro, técnico em enfermagem, condutor, telefonista, operador de rádio e administrativo. O serviço atendia unicamente a cidade de Poços de Caldas, prestando por mês uma média de 1000 atendimentos com saída de viatura, além de cerca de 1500 orientações. A maior demanda estava relacionada a atendimento clínico de adultos (Desvio-Padrão - DP=1 100/mês), seguido por atendimento a politraumatizados (DP=310/mês).

A amostra foi composta por 63 profissionais dos diferentes cargos, distribuídos nos diversos turnos de trabalho, com jornada de 6 horas (manhã, tarde e noite), 8 horas (administrativo) e 12 horas (diurno e noturno). A faixa etária dos participantes esteve compreendida entre 20 e 57 anos, com idade média de 32,6 anos (DP=8,65) e predomínio do sexo masculino (60,3\%).

Os instrumentos utilizados para a coleta dos dados foram Ficha de caracterização sociodemográfica e Inventário de Sintomas de Stress de Lipp para Adultos (ISSL) (Lipp, 2000).

Os aspectos éticos foram respeitados, conforme a Resolução 196/96 do Conselho Nacional de Saúde, referente às recomendações para pesquisas com seres humanos. Além disso, antes do início da coleta de dados, o projeto foi submetido a análise do Comitê de Ética em Pesquisa da Faculdade de Ciências Médicas da Unicamp, onde foi aprovado mediante Parecer no 190/2009.

\section{Procedimentos}

Inicialmente foi distribuído a todos os funcionários da equipe um documento explicando sobre a pesquisa na unidade e sobre a importância da participação de cada um. Após confirmação da participação de cada sujeito, foi entregue e assinado um Termo de Compromisso Livre e Esclarecido.

Em seguida, teve início a coleta dos dados, com o preenchimento da ficha de caracterização sociodemográfica e, posteriormente, do ISSL. Os dados foram coletados no mês de abril de 2009, durante o turno de trabalho dos funcionários, sendo que o próprio pesquisador entregou os instrumentos, fornecendo orientações sobre o preenchimento e esclarecendo as dúvidas encontradas.

A análise estatística dos dados obtidos na pesquisa foi feita através do programa SPSS 16, com os 
testes Qui-Quadrado e Teste Exato de Fisher, e apresentada na forma descritiva e em tabelas de frequência das variáveis categóricas e contínuas. O nível de significância adotado para os testes estatísticos foi de $5 \%$, ou seja, $p<0,05$.

A correção e interpretação dos ISSL foram feitas por uma psicóloga, conforme solicita o Conselho Federal de Psicologia.

\section{Resultados}

Diante da formação interdisciplinar da equipe e da dinâmica do trabalho, optou-se por efetuar a análise dos dados como um todo. Dos 63 sujeitos que participaram da pesquisa, houve predomínio do sexo masculino (60,3\%), com idade entre 20 e 39 anos $(81,0 \%)$ e média de 32,6 anos (DP=8,65).

Quanto ao estado civil, 36,5\% eram solteiros e 46,0\% casados. Em relação à escolaridade, 58,7\% tinham ensino médio completo e 33,3\% ensino superior completo. Observa-se que essa incidência é devida à existência de diversos cargos em que não há exigência de maior nível de escolaridade, como técnico em enfermagem, condutor de veículo, telefonista e operador de rádio.

Quanto ao cargo ocupado, verificou-se maior frequência de condutores de veículos $(25,4 \%)$ e médicos (20,6\%). Quando investigado o tempo de trabalho na unidade, obteve-se que 63,5\% dos sujeitos trabalhavam há mais de 13 meses, com média de 20,6 meses (DP=9,9), ou seja, a grande maioria trabalhava na instituição desde sua implantação. Na distribuição dos sujeitos por turno de trabalho, observou-se que 28,6\% estavam alocados no turno diurno, e 27,0\% no turno noturno, ambos com jornadas de 12 horas.

Quando investigada a presença de outro trabalho, obteve-se que 50,8\% não o possuíam, e 49,2\% que tinham outro trabalho. Quanto a terem outra atividade, obteve-se que 66,7\% não a tinham.

Os sujeitos com condução própria correspondiam a 69,8\% da amostra, e os que residiam na mesma cidade, a 92,1\% (Tabela 1).

Pode-se observar que, na amostra de 63 sujeitos, 20 (31,7\%) apresentavam sintomas de stress e 68,3\% não apresentavam sintomas de stress.
Quanto à fase do stress avaliada através do ISSL, obteve-se como resultado que 30,2\% dos sujeitos com stress encontravam-se na fase de resistência, e 1,6\% na fase de alerta. Com relação à fase de quase exaustão e exaustão, os resultados não demonstraram sujeitos com essa classificação.

Dentre os sujeitos com stress, quanto à prevalência dos sintomas, foi verificado que 14,3\% apresentavam sintomas físicos; $12,7 \%$, sintomas psicológicos; e 4,8\%, tanto sintomas físicos quanto psicológicos.

Na ocorrência do stress de acordo com o sexo, os homens apresentaram maior incidência (17,5\%), porém não significativa estatisticamente.

Quando verificada a ocorrência do stress de acordo com a idade, observou-se maior incidência entre os sujeitos de 30 a 39 anos (12,7\%).

Quanto à ocorrência do stress relacionado ao estado civil, não foi observada diferença entre sujeitos casados e solteiros, tendo-se obtido respectivamente $14,3 \%$ e 12,7\% de incidência.

Dentre os sujeitos com stress, observou-se maior incidência naqueles que tinham ensino médio completo, como os técnicos de enfermagem (9,5\%) e os condutores de veículo, que executam tarefas sabidamente estressantes, conforme evidenciado em diversos estudos (Aguiar et al., 2000; Calderero, Miasso \& Webster, 2008; Cristina, Dalri, Cyrillo, Salki \& Veiga, 2008; Malagris \& Fiorito, 2006).

Quanto à presença do stress relacionado ao tempo de trabalho na unidade, dentre os sujeitos com menos de 6 meses de trabalho, nenhum apresentou stress, e dentre os que trabalhavam há mais de 13 meses, 22,2\% apresentavam sintomas de stress. Quanto à incidência de stress relacionado ao turno de trabalho, foi verificado stress em 12,7\% dos sujeitos do turno diurno, enquanto se verificou ausência de stress em 20,6\% dos sujeitos do turno noturno (Tabela 2). Isso pode ser justificado pela maior ocorrência de chamados durante o turno diurno, o que exporia esses profissionais a uma maior demanda de atividades estressantes.

Quando investigada a incidência do stress nos sujeitos que tinham outro trabalho, foi observada distribuição homogênea, sendo que, dentre os sujeitos com stress, 16,0\% tinham um segundo emprego e outros $16,0 \%$ não. 
Tabela 1. Características gerais da amostra ( $n=63)$ estudada. Poços de Caldas (MG), 2009.

\begin{tabular}{|c|c|c|c|c|}
\hline Variável & Frequência & $\%$ & Média & Desvio-Padrão \\
\hline \multicolumn{5}{|l|}{ Sexo } \\
\hline Masculino & 38 & 60,3 & & \\
\hline Feminino & 25 & 39,7 & & \\
\hline Idade & & & 32,6 & 8,65 \\
\hline $20-29$ anos & 25 & 39,7 & & \\
\hline $30-39$ anos & 26 & 41,3 & & \\
\hline $40-49$ anos & 8 & 12,7 & & \\
\hline $50-59$ anos & 4 & 6,3 & & \\
\hline \multicolumn{5}{|l|}{ Estado civil } \\
\hline Solteiro & 23 & 36,5 & & \\
\hline Casado & 29 & 46,0 & & \\
\hline Divorciado & 5 & 7,9 & & \\
\hline Amasiado & 5 & 7,9 & & \\
\hline Outros & 1 & 1,0 & & \\
\hline \multicolumn{5}{|l|}{ Escolaridade } \\
\hline Fundamental completo & 3 & 4,8 & & \\
\hline Médio completo & 37 & 58,7 & & \\
\hline Superior completo & 21 & 33,3 & & \\
\hline Pós-graduação & 2 & 3,2 & & \\
\hline \multicolumn{5}{|l|}{ Cargo } \\
\hline Médico & 13 & 20,6 & & \\
\hline Enfermeiro & 5 & 7,9 & & \\
\hline Técnico de enfermagem & 11 & 17,5 & & \\
\hline Condutor & 16 & 25,4 & & \\
\hline Telefonista & 10 & 15,9 & & \\
\hline Operador de rádio & 6 & 9,5 & & \\
\hline Administrativo & 2 & 3,2 & & \\
\hline Tempo de trabalho no SAMU (meses) & & & 20,6 & 9,9 \\
\hline 4 a 6 meses & 2 & 3,2 & & \\
\hline 7 a 12 meses & 21 & 33,3 & & \\
\hline$>13$ meses & 40 & 63,5 & & \\
\hline \multicolumn{5}{|l|}{ Turno de trabalho } \\
\hline Manhã & 4 & 6,3 & & \\
\hline Tarde & 7 & 11,1 & & \\
\hline Noite & 8 & 12,7 & & \\
\hline Diurno & 18 & 28,6 & & \\
\hline Noturno & 17 & 27,0 & & \\
\hline Diurno e noturno & 9 & 14,3 & & \\
\hline \multicolumn{5}{|l|}{ Outro trabalho } \\
\hline Sim & 31 & 49,2 & & \\
\hline Não & 32 & 50,8 & & \\
\hline \multicolumn{5}{|l|}{ Outra atividade } \\
\hline Sim & 21 & 33,3 & & \\
\hline Não & 42 & 66,7 & & \\
\hline \multicolumn{5}{|l|}{ Condução própria } \\
\hline Sim & 44 & 69,8 & & \\
\hline Não & 19 & 30,2 & & \\
\hline \multicolumn{5}{|l|}{ Reside na mesma cidade } \\
\hline Sim & 58 & 92,1 & & \\
\hline Não & 5 & 7,9 & & \\
\hline
\end{tabular}


Tabela 2. Incidência de stress relacionado ao turno de trabalho. Poços de Caldas (MG), 2009.

\begin{tabular}{|c|c|c|c|c|c|}
\hline \multirow[b]{2}{*}{ Turno } & \multicolumn{2}{|c|}{ Stress - Sim } & \multicolumn{2}{|c|}{ Stress - Não } & \multirow[t]{2}{*}{ Total } \\
\hline & Frequência & $\%$ & Frequência & $\%$ & \\
\hline Manhã & 0 & 0 & 4 & 6,4 & 4 \\
\hline Tarde & 5 & 7,9 & 2 & 3,2 & 7 \\
\hline Noite & 1 & 1,6 & 7 & 11,1 & 8 \\
\hline Diurno & 8 & 12,7 & 10 & 16,0 & 18 \\
\hline Noturno & 4 & 6,4 & 13 & 20,6 & 17 \\
\hline Diurno/Noturno & 2 & 3,2 & 7 & 11,1 & 9 \\
\hline Total & 20 & & 43 & & 63 \\
\hline
\end{tabular}

Teste Qui-Quadrado: $p=0,061$.

Tabela 3. Incidência de stress relacionado a ter outra atividade. Poços de Caldas (MG), 2009.

\begin{tabular}{|c|c|c|c|c|c|}
\hline \multirow[b]{2}{*}{ Outra atividade } & \multicolumn{2}{|c|}{ Stress - Sim } & \multicolumn{2}{|c|}{ Stress - Não } & \multirow[t]{2}{*}{ Total } \\
\hline & Frequência & $\%$ & Frequência & $\%$ & \\
\hline Sim & 11 & 17,5 & 10 & 16,0 & 21 \\
\hline Não & 9 & 14,3 & 33 & 52,4 & 42 \\
\hline Total & 20 & & 43 & & 63 \\
\hline
\end{tabular}

Teste Exato de Fisher: $p=0,015$.

Tabela 4. Incidência de stress relacionado a possuir condução própria. Poços de Caldas (MG), 2009.

\begin{tabular}{|c|c|c|c|c|c|}
\hline \multirow[b]{2}{*}{ Condição própria } & \multicolumn{2}{|c|}{ Stress - Sim } & \multicolumn{2}{|c|}{ Stress - Não } & \multirow[t]{2}{*}{ Total } \\
\hline & Frequência & $\%$ & Frequência & $\%$ & \\
\hline Sim & 11 & 17,5 & 33 & 52,4 & 44 \\
\hline Não & 9 & 14,3 & 10 & 16,0 & 19 \\
\hline Total & 20 & & 43 & & 63 \\
\hline
\end{tabular}

Teste Exato de Fisher: $p=0,074$.

Quanto à ocorrência do stress nos sujeitos que tinham outro trabalho, relacionado ao turno na outra instituição, foi observada maior incidência entre os sujeitos que trabalhavam em turnos de 12 horas $(4,8 \%)$ e em horário administrativo (4,8\%).

Na Tabela 3 pode-se observar a avaliação da incidência do stress nos sujeitos que tinham outra atividade, sendo que $52,4 \%$ dos sujeitos que não tinham outra ativdade não apresentavam sintomas de stress, resultado estatisticamente significativo $(p=0,015)$.

Quando avaliada a incidência do stress relacionado à existência de condução própria, obteve-se que $52,4 \%$ dos sujeitos que a possuíam não apresentavam sinais de stress, conforme mostra a Tabela 4.

Quanto à ocorrência de stress relacionado à residência na mesma cidade do trabalho, obteve-se que
65,1\% dos sujeitos nessa situação não apresentavam stress. O fato de a maioria dos sujeitos possuírem condução própria e residirem na mesma cidade provavelmente ameniza a ocorrência do stress advindo do deslocamento da residência até a unidade de trabalho.

\section{Discussão}

Os dados encontrados na presente pesquisa mostram sujeitos jovens, com idade de significativa capacidade produtiva, provavelmente devido às características das atividades executadas nos atendimentos. Foi verificado também que grande parte dos profissionais tem apenas um único trabalho, achado contrário ao que tem sido encontrado em outras pesquisas, nas quais a maioria dos sujeitos exerce dupla jornada e 
atribuem isso à necessidade econômica, devido aos baixos salários dos profissionais que atuam na área da saúde (Calderero et al., 2008; Pafaro \& De Martino, 2004; Vieira, 2001). Porém, os dados obtidos corroboram os encontrados na pesquisa de Ferreira e De Martino (2009), em que a maioria dos profissionais de enfermagem não tinha outro emprego.

Para explicar a baixa prevalência de stress na amostra, este estudo acompanha a sugestão de Aguiar et al. (2000), de que é possível que os sujeitos já tenham desenvolvido mecanismos de enfrentamento. Além disso, outro fator que pode justificar o achado é o fato de os profissionais terem desejo expresso de atuar nessa área, por poderem exercer sua autonomia e sentir que têm certo controle sobre sua vida. Essa percepção de controle pode ser caracterizada como um fator protetor para o stress (Aguiar et al., 2000; Calderero et al., 2008).

Quanto à fase do stress encontrada na amostra, predominou a fase de resistência, sendo esse dado também contrário ao de outros estudos, em que houve maior incidência de stress entre os profissionais que atuam em emergência e atendimento pré-hospitalar. Esses trabalhos apontaram como fatores estressantes a violência a que o profissional pode estar exposto, além das características inerentes do trabalho, como lidar com a dor, o sofrimento e a morte (Aguiar et al., 2000; Calderero et al., 2008; Cristina et al., 2008, Encalada, Zegarra, Malca \& Tello, 2007). Segundo Malagris e Fiorito (2006), o alto índice de sujeitos na fase de resistência parece revelar um desgaste acumulado ao longo de algum tempo, relacionado ao tipo de atividade profissional desenvolvida.

Foi verificada prevalência de stress nos sujeitos do sexo masculino, sendo esse achado pouco frequente, pois tem sido observada maior ocorrência de stress nas mulheres, podendo isso estar associado à dupla ou até tripla jornada caracterizada pelas atividades domésticas, que, na sociedade brasileira, na maioria das vezes, é um trabalho desvalorizado e atribuído à esfera de competência da mulher (Calderero et al., 2008; Ferreira \& De Martino, 2009).

O stress foi observado com maior frequência entre sujeitos que tinham maior tempo de serviço na unidade, sendo que isso se deve possivelmente ao fato de estarem eles constantemente expostos a agentes estressores 206 durante a jornada de trabalho. Assim, são repetidamente obrigados a entrar na primeira fase do stress, podendo ainda, com o passar do tempo, evoluir para as demais fases (Aguiar et al., 2000).

Pode-se verificar, de acordo com diversos estudos sobre atendimento de urgência e pré-hospitalar, que essas atividades são consideradas potencialmente estressoras, dado confirmado pela alta incidência de stress nos indivíduos que atuam na área. Conforme apontam A.A. Soerensen, Moriya, R. Soerensen e Robazi (2008), os profissionais que atuam em serviço de APH móvel enfrentam inúmeras dificuldades, como a grande exposição aos riscos ocupacionais durante o atendimento às vítimas e/ou a escassez de literatura que possa servir de embasamento para a resolutividade de tais deficiências.

Além disso, várias são as características gerais exigidas para a execução desse trabalho, como experiência profissional e habilidade, além de outras específicas, como a formação voltada a esse tipo de atendimento, a capacidade física de lidar com o stress, tomar decisões imediatas, definir prioridades e trabalhar em equipe com outros profissionais, como policiais e companhia de energia elétrica, entre outros (Thomaz \& Lima, 2000).

Estudo conduzido por Murta e Tróccoli (2007) sobre os efeitos de intervenção para reduzir o stress ocupacional, com base na avaliação de necessidades de bombeiros, indicou a existência de vários estressores ocupacionais, relacionados principalmente à organização e às condições de trabalho da equipe.

Porém, tal fato não foi observado neste estudo, em que houve baixa ocorrência de stress, com os indivíduos estando, em sua maioria, na fase de resistência, não sendo encontrados sujeitos na fase de quase exaustão e exaustão. Esses dados, concordando com Aguiar et al. (2000), conduzem à conclusão de que os indivíduos, de maneira consciente ou inconsciente, utilizam-se de mecanismos cognitivos ou comportamentais a fim de minimizar o stress e manter o equilíbrio interno.

É ainda importante a consideração de Sanzovo e Coelho (2007) de que nem sempre as dimensões da reação de stress são proporcionais às dos agentes estressores. Isso pode estar relacionado à singularidade da história de vida da cada indivíduo, eà maneira como se interpreta a ação dos estressores. 
Além disso, conforme citado anteriormente, o fato de o profissional optar por exercer suas atividades nesse local de trabalho favorece o exercício de sua autonomia, permitindo sentir controle sobre sua vida, podendo esse sentimento se tornar um fator protetor contra o stress. Ainda é possível afirmar, de acordo com Calderero et al. (2008) e com Cristina et al. (2008), que o fato de o profissional poder escolher seu local de trabalho pode implicar maior satisfação e motivação, o que poderia também culminar em menor ocorrência de stress.

\section{Considerações Finais}

No presente estudo, foi observada baixa incidência do stress em equipe multiprofissional de atendimento pré-hospitalar, fato esse que não tem sido comumente relatado em outros estudos, que apontam elevados níveis de stress, devidos principalmente às características de imprevisibilidade desse tipo de atividade. Sugere-se a realização de novos estudos com esse grupo ocupacional, com amostras maiores, em localidades com outras características de atendimento, para melhor identificar o quanto esses achados podem ser extrapolados para outras equipes de APH móvel, ou se foram idiossincráticos dessa amostra. Essas pesquisas favorecem o aumento da produção do conhecimento sobre o tema, fornecendo subsídios para o desenvolvimento de políticas públicas de saúde, com atenção às estratégias de proteção aos profissionais que atuam no atendimento pré-hospitalar móvel, garantindo melhor adequação frente às exigências do trabalho.

\section{Referências}

Aguiar, K. N., Silva, A. L. C., Faria, C. R., Lima, F. V., Souza, P. R., \& Stacciarini, L. M. R. (2000). O estresse em uma equipe militar de resgate pré-hospitalar. Revista Eletrônica de Enfermagem, 2 (2). Recuperado em maio 20, 2009, disponível em <http://www.fen.ufg.br/revista/revista2_2/ stress $>$.

Bachion, M. M., Peres, A.S., Belisário, V. L., \& Carvalho, E. C. (1998). Estresse, ansiedade e coping: uma revisão dos conceitos, medidas e estratégias de intervenção voltadas para a prática de enfermagem. Revista Mineira de Enfermagem, 2 (1), 33-39.

Brasil. Ministério da Saúde. (2002, 12 de novembro). Portaria GM/MS n. 2048, de 5 de novembro de 2002. Aprova o regulamento técnico dos sistemas estaduais de urgência e emergência. Diário Oficial da União, Brasília, Seção 1, pp.32-54.
Brasil. Ministério da Saúde. (2003, 6 de outubro). Portaria GM/MS n. 1864, de 20 de setembro de 2003. Institui o componente pré-hospitalar móvel da Política Nacional de Atenção às Urgências, por intermédio da implantação do serviço de Atendimento Móvel de Urgência em municípios e regiões de todo o território brasileiro: SAMU-192. Diário Oficial da União, Brasília. Seção 1, pp. 57-9.

Brasil. Ministério da Saúde. (2004a). Regulamento técnico dos sistemas de urgência e emergência. Brasília: Ministério da Saúde.

Brasil. Ministério da Saúde. (2004b). SAMU-192: o que é o SAMU? Recuperado em fevereiro 26, 2009, disponível em <http://www.saude.gov.br/samu-programa-nacional>.

Brasil. Ministério da Saúde. (2006). Política nacional de atenção às urgências (3a ed). Brasília: Ministério da Saúde.

Calais, S. L. (2003). Diferenças entre homens e mulheres na vulnerabilidade ao stress. In M. E. N. Lipp (Ed.), Mecanismos neuropsicofisiológicos do stress: teoria e aplicações clínicas (pp.87-90) São Paulo: Casa do Psicólogo.

Calderero, A. R. L., Miasso, A. I., \& Webster, C. M. C. (2008). Estresse e estratégias de enfrentamento em uma equipe de enfermagem de pronto atendimento. Revista Eletrônica de Enfermagem, 10 (1): 51-62. Recuperado em junho 5, 2009, disponível em <http://www.fen.ufg.br/ revista/v10/n1/v10n1a05>.

Camponovo Méier, O., \& Morín Imbert, P. (2000). Síndrome de burnout en el personal de salud de un hospital público de la ciudad de Rosario. Investigación en Salud, 3 (1-2), 73-93.

Cox, T. (1987). Stress, coping and problem solving. Work and Stress, 1 (1), 5-14.

Cristina, J. A., Dalri, M. C. B., Cyrillo, R. M. Z., Salki, T., \& Veiga, E. V. (2008). Vivências de uma equipe multiprofissional de atendimento pré-hospitalar móvel em suporte avançado de vida na assistência ao adulto em situação de parada cárdiorespiratória. Revista Ciência y Enfermeria, 14 (2), 97-105.

De Gasperi, P., \& Radünz, V. (2006). Cuidar de si: essencial para enfermeiros. REME: Revista Mineira de Enfermagem, $10(1), 82-7$

De Martino, M. M. F., \& Cipolla-Neto, J. (1999). Repercussões do ciclo vigília-sono e o trabalho em turnos de enfermeiras. Revista de Ciências Médicas, 8 (3), 81-4.

Encalada, A. M., Zegarra, R. O., Malca, A. M. U., \& Tello, M. V. (2007). Factores que desencadenan el estrés y sus consecuencias em el desempeño laboral en emergência. Revista Peruana de Obstetricia y Enfermaria, 3 (1), 50-57.

Ferreira, L.R. C., \& De Martino, M. M. F. (2009). Stress no cotidiano da equipe de enfermagem e sua correlação com o cronótipo. Estudos de Psicologia (Campinas), 26 (1), 65-72. doi: 10.1590/S0103-166X2009000100007.

França, A. C. L., \& Rodrigues, A. L. (2002). Stress e trabalho: uma abordagem psicossomática. São Paulo: Atlas.

Glassman, W. E., \& Hadad, M. (2006). Psicologia: abordagens atuais ( $4^{\mathrm{a}}$ ed.). Porto Alegre: Artmed. 
Jacques, M. G. (2004) Abordagens. In L. B. M. Guimarães. Ergonomia: tópicos especiais, qualidade de vida no trabalho, psicologia e trabalho (pp.2.1-4). Porto Alegre: UFRGS.

Lazarus, R. S., \& Folkman, S. (1984). Stress, appraisal \& coping. Nova York: Springer.

Lipp, M. E. N. (Org). (1996). Pesquisas sobre stress no Brasil: saúde, ocupações e grupos de risco. Campinas: Papirus.

Lipp, M. E. N. (2000). Inventário de sintomas de stress para adultos de Lipp. São Paulo: Casa do Psicólogo.

Lipp, M. E. N. (2003a). Mecanismos neuropsicofisiológicos do stress: teoria e aplicações clínicas. São Paulo: Casa do Psicólogo.

Lipp, M. E. N. (2003b). O modelo quadrifásico do stress. In M. E. N. Lipp (Ed.), Mecanismos neuropsicofisiológicos do stress: teoria e aplicações clínicas (pp.17-21) São Paulo: Casa do Psicólogo.

Lipp, M. E. N., \& Tanganelli, M. S. (2002). Stress e qualidade de vida em magistrados da justiça do trabalho: diferença entre homens e mulheres. Psicologia: Reflexão e Crítica, 15 (3), 537-548

Malagris, L. E. N., \& Fiorito, A. C. C. (2006). Avaliação do nível de stress de técnicos da área de saúde. Estudos de Psicologia (Campinas), 23 (4), 391-8. doi: 10.1590/S0103-166X2 006000400007

Martins, L. M. M., Bronzatti, J. A. G., Vieira, C. S. C. A., Parra, S. H. B., \& Silva, Y. B. (2000). Agentes estressores no trabalho e sugestões para amenizá-los: opiniões de enfermeiros de pós-graduação. Revista da Escola de Enfermagem USP, 34 (1), 52-8.

McGowan, J., Gardner, D., \& Fletcher, R. (2006). Positive and negative affective outcomes of occupational stress. New Zeeland Journal of Psychology, 35 (2), 92-98.

Murta, S. G., \& Tróccoli, B. T. (2007). Stress ocupacional em bombeiros: efeitos de intervenção baseada em avaliação de necessidades. Estudos de Psicologia (Campinas), 24 (1), 41-51. doi: 10.1590/S0103-166X2007000100005.

Paschoal, T., \& Tamayo, A. (2004). Validação da escala de estresse no trabalho. Estudos de Psicologia (Natal), 9 (1), 45-52.

Pafaro, R. C., \& De Martino, M. M. F. (2004). Estudo do estresse do enfermeiro com dupla jornada de trabalho em um hospital de oncologia pediátrica da Campinas. Revista da Escola de Enfermagem USP, 38 (2), 152-60.

Santos, J. M., Oliveira, E. B., \& Moreira, A. C. (2006). Estresse, fator de risco para a saúde do enfermeiro em centro de terapia intensiva. Revista de Enfermagem UERJ, 14 (4), 580-5.

Sanzovo, C. E., \& Coelho, M. E. C. (2007). Estressores e estratégias de coping em uma amostra de psicólogos clínicos. Estudos de Psicologia (Campinas), 24 (2), 227-238. doi: 10.1590/S0103-166X2007000200009.

Sears, L. E., Murphy, L. A., Sinclair, R. R., Davidson, S. B., \& Wang, M. (2008) Insufficient staffing: missed breaks, overtime and safe nursing care delivery. In Conference Abstracts of Work, Stress and Health - Health and Safe Work Throught Research, Practice and Partnerships. Washington, DC: NIOSH/SOHP/APA.

Selye, H. (1954). Stress, a tensão da vida. São Paulo: Ibrasa.

Silva, E. A. T., \& Martinez, A. (2005). Diferença em nível de stress em duas amostras: capital e interior de São Paulo. Estudos de Psicologia (Campinas), 22 (1), 53-61. doi: 10.15 90/S0103-166X200500100007.

Soerensen, A. A., Moriya, T. M., Soerensen, R., \& Robazzi, M. L. C. C. (2008). Atendimento pré-hospitalar móvel: fatores de riscos ocupacionais. Revista Enfermagem UERJ, 16 (2), 187-92.

Stacciarini, J. M. R., \& Tróccoli, B. T. (2000). O Estresse na atividade ocupacional do enfermeiro. Revista Latino-Americana de Enfermagem, 9 (2), 17-25.

Thomaz, R. R., \& Lima, F. V. (2000). Atuação do enfermeiro no atendimento Pré-Hospitalar na cidade de São Paulo. Acta Paulista de Enfermagem, 13 (3), 59-65.

Vieira, L. C. (2001). Estresse ocupacional em enfermeiros de um hospital universitário da cidade de Campinas. Dissertação de mestrado não-publicada, Faculdade de Ciências Médicas, Universidade Estadual de Campinas.

Zaldúa, G., \& Lodieu, M. (2000). El burnout. La salud de los trabajadores de la salud. Revista del Instituto de Investigaciones de la Facultad de Psicología, 5 (1), 151-169.

Recebido em: 14/12/2009

Versão final reapresentada em: 19/7/2010

Aprovado em: 6/10/2010 